\title{
Revenue Sharing in Hierarchical Organizations: A New Interpretation of the Generalized Banzhaf Value
}

\author{
Roland Pongou ${ }^{1}$, Bertrand Tchantcho ${ }^{2,3}$, Narcisse Tedjeugang ${ }^{2}$ \\ ${ }^{1}$ Department of Economics, University of Ottawa, Ottawa, Canada \\ ${ }^{2}$ École Normale Supérieure, Yaoundé, Cameroon \\ ${ }^{3}$ Université de Cergy Pontoise, Cergy-Pontoise, France \\ Email: rpongou@uottawa.ca, btchantcho@yahoo.fr, tedjeugang@yahoo.fr
}

Received February 24, 2012; revised March 23, 2012; accepted April 19, 2012

\begin{abstract}
This paper examines the distribution of earnings in a new model of hierarchical multi-task organizations. Each such organization is defined by a finite set of workers and tasks together with a production function that maps each allocation of workers to the tasks into an aggregate output. Tasks are ordered in degree of importance so that aggregate output increases when a worker climbs up the organization ladder. We show that the generalized Banzhaf value proposed by Freixas [1] can be used as a theory of revenue sharing in such organizations, and provide a new interpretation and formulation of this sharing rule, proving that a worker's pay is proportional to the difference between his marginal productivity at the top level and at the bottom level of the hierarchy summed over all the possible configurations of the organization. This new formulation also facilitates computation.
\end{abstract}

Keywords: Hierarchical Organizations; Earnings; Marginal Productivity; Generalized Banzhaf Value

\section{Introduction}

This paper studies the distribution of earnings in a new model of hierarchical organizations introduced in Pongou et al. [2]. Each such organization is defined by a finite set of workers and tasks, and a production function that maps each allocation of workers to the tasks into an aggregate output. Tasks are ordered in degree of importance so that aggregate output increases when a worker climbs up the organization ladder. Assuming that the organization is competitive, output converts directly into revenue.

We adapt the generalized Banzhaf value proposed by Freixas [1] as a theory of revenue sharing in such organizations. This notion is an extension of the concept known as the Banzhaf-Coleman value. It was independently proposed by Banzhaf [3] and Coleman [4] as a measure of the ability of a voter to affect the outcome of a voting game. An axiomatic study of this value was conducted by Dubey and Shapley [5] and Laruelle and Valenciano [6]. Important applications of the Banzhaf value to corporations and corporate governance can be found in Leech [7,8]. Feltkamp [9] generalizes the axiomatic approach to any transferable utility cooperative game. In the context of the firm, a cooperative game can be viewed as a hierarchical organization that has only two levels. In an attempt to generalize this notion, Freixas and Zwicker [10] introduce the concept of $(j, k)$ simple games. A $(j, k)$ simple game can be interpreted as a hierarchical organization that has $j$ levels of hierarchy, and is equipped with a real-valued production function whose range has cardinality $k$. In our model of a hierarchical organization, $k$ is not fixed, but is endogenously determined by the production function. Freixas [1] generalizes the relative Banzhaf value to this class of games. Diffo Lambo and Moulen [11] and Freixas et al. [12] study this value in relation to other theories within the class of $(2,2)$ simple games, and Tchantcho et al. [13] and Pongou et al. [14] extend this analysis to the class of $(3,2)$ simple games.

A new formulation and interpretation of the generalized Banzhaf value is provided. It proves that in a hierarchical organization, the Banzhaf value of a worker is proportional to the difference between his marginal productivity at the top level and at the bottom level of the hierarchy summed over all the possible configurations of the organization. This new formulation also makes this notion very easy to compute.

The remainder of the paper is organized as follows. Section 2 defines hierarchical organizations. In Section 3, a new formulation of the generalized Banzhaf value is provided. Section 4 concludes.

\section{Hierarchical Organizations}

An organization is a list $(N, T, V)$ where $N$ is a non- 
empty finite set of workers, $T=\left\{T_{1}, \cdots, T_{j}\right\}$ a finite collection of finite sets of tasks with cardinility $|T|=j \geq 2$, and $V$ a production function that maps each allocation of workers to the tasks into a real number which measures the aggregate output or productivity of the organization under that particular configuration (Pongou et al. [2]). For every $i=1, \cdots j, T_{i}$ is a set of identical tasks. Denote by $t_{i}$ a representative task of $T_{i}$. An allocation of workers to the tasks is a $j$-partition $A=\left({ }_{1} A, \cdots,{ }_{j} A\right)$ of $N$, where ${ }_{i} A$ is the set of workers assigned to type $t_{i}$ tasks (or simply to task $t_{i}$ ). Under an allocation $A=\left({ }_{1} A, \cdots,{ }_{j} A\right),{ }_{i} A$ may be empty for any $i=1, \cdots j$, which would mean that no worker has been assigned to task $t_{i}$. The set of all possible allocations is denoted $\mathcal{N}^{j}$.

In a hierarchical organization, tasks are ordered in degree of importance: $t_{1}$ is the most important task, $t_{2}$ the second most important task, and so on. A typical example is a military organization where each higherranking member is more competent and has more important tasks than his subordinates. A hierarchical organization is said to be monotonic if aggregate output increases as a worker moves from a less important task to a more important one. The concept of monotonicity is formalized below.

Let $A=\left({ }_{1} A, \cdots,{ }_{j} A\right)$ be an ordered $j$-partition of $N$. For any $l \in\{1,2, \cdots, j\}$, denote by $A^{l}={ }_{1} A \cup \cdots \cup, A$ the set of workers executing a task at least as important as task $t_{l}$. Let $B=\left({ }_{1} B, \cdots,{ }_{j} B\right)$ be an ordered $j$-partition of $N$ such that $A^{l} \subseteq B^{l}$ for any $l=1, \cdots j$. That is, $B$ is obtained from $A$ by moving some workers to more important tasks. This is denoted by $A \subseteq{ }^{j} B$. A multi-task organization $(N, T, V)$ is said to be monotonic if for any ordered $j$-partitions $A, B \in \mathcal{N}^{j}$ such that $A \subseteq^{j} B, V(B) \geq V(A)^{1}$.

\section{The Generalized Banzhaf Value and a New Formulation}

The generalized Banzhaf value developed by Freixas [1] is adapted to our framework as follows. Let $(N, T, V)$ be a monotonic hierarchical organization, and $\left\{v_{1}, v_{2}, \ldots, v_{k}\right\}$ the range of $V$. Without loss of generality, assume $v_{1}>v_{2}>\cdots>v_{k}$. Let $A$ be an allocation of workers and $p$ a worker. Denote by $\alpha(p, A)$ the task assigned to $p$ in the allocation $A$, and by $A_{p \downarrow}$ the allocation obtained from $A$ by moving $p$ one level down the organization ladder :

$$
\alpha\left(p, A_{p \downarrow}\right)=\alpha(p, A)+1,
$$

\footnotetext{
${ }^{1} \mathrm{~A}$ monotonic hierarchical organization as defined here can be viewed as a reinterpretation of the notion of $(j, k)$ simple games (Freixas and Zwicker [10]). Note, however, that not all hierarchical organizations are monotonic (Pongou et al. [2]).
}

$$
\alpha\left(q, A_{p \downarrow}\right)=\alpha(q, A) \quad \forall q \in N, q \neq p .
$$

Let $l, m \in\{1,2, \cdots, k\}$ be such that $1 \leq l<m \leq k$. $p$ is said to be $(l, m)$-critical in $A$ if:

$$
v_{l}=V(A)>V\left(A_{p \downarrow}\right)=v_{m} .
$$

Denote by $\eta_{p}^{l, m}(V)$ the number of allocations in which $p$ is $(l, m)$-critical, and by ${ }_{i} \eta_{p}^{l, m}(V)$ the number of allocations $A=\left({ }_{1} A, \ldots,{ }_{j} A\right)$ in which $p$ is $(l, m)$-critical and $p \in{ }_{i} A(1 \leq i \leq j-1)$. It follows that:

$$
\eta_{p}^{l, m}(V)=\sum_{i=1}^{j-1}{ }_{i} \eta_{p}^{l, m}(V) .
$$

The Banzhaf value is defined below.

Definition 1. Let $(N, T, V)$ be a monotonic hierarchical organization, and $p \in N$ a worker.

1) The Banzhaf score of $p$ is:

$$
\eta_{p}(V)=\sum_{m=2}^{k} \sum_{l=1}^{m-1}\left(v_{l}-v_{m}\right) \eta_{p}^{l, m}(V)
$$

2) The normalized Banzhaf index of $p$ is:

$$
\beta_{p}(V)=\frac{\eta_{p}(V)}{\sum_{i \in \mathcal{N}^{j}} \eta_{i}(V)},
$$

The Banzhaf index of a worker measures his earnings in terms of the proportion of the organization's revenue he is expected to obtain. Our goal is to give a new interpretation of this sharing rule. Let $A$ be an allocation of workers and $p \in N$ a worker. Denote by $A_{p}^{1}$ the allocation obtained by moving $p$ from his position in $A$ up to the top level of the organization. Similarly, denote by $A_{p}^{j}$ the allocation obtained by moving $p$ from his position in $A$ down to the bottom level of the organization. We show below that the Banzhaf score of $p$ is proportional to the difference between his marginal productivity at the top level of the organization $\left(V\left(A_{p}^{1}\right)-V(A)\right)$ and his marginal productivity at the bottom level $\left(V\left(A_{p}^{j}\right)-V(A)\right)$ summed over all the possible worker allocations.

Theorem 1. Let $(N, T, V)$ be a monotonic hierarchical organization, and $p \in N$ a worker. The Banzhaf score of $p$ is given by:

$$
\eta_{p}(V)=\frac{1}{j} \sum_{A \in \mathcal{N}^{j}} V\left(A_{p}^{1}\right)-V\left(A_{p}^{j}\right) .
$$

The definition below will be needed in the proof of Theorem 1.

Definition 2. Let $A$ and $B$ be two j-partitions of $N$ and $p$ a worker.

$B$ is said to be $p$-equivalent to $A$ if $\forall q \in N, \quad q \neq p$, $\alpha(q, B)=\alpha(q, A)$.

It is obvious that the relation defined above is an equivalence relation. The number of elements in each equi- 
valence class is $j$, and since the number of $j$-partitions is $j^{n}$, there are exactly $j^{n-1}$ equivalence classes. We denote these equivalence classes by $\mathcal{N}_{p}^{j}(t)$, $t=1,2, \cdots, j^{n-1}$.

Theorem 1 is proved below.

Proof. It is the case that the Banzhaf score of a worker $p$ is the number of $j$-partitions in which $p$ is $(l, m)$ critical, so that:

$$
\begin{aligned}
\eta_{p}(V) & =\sum_{A \in \mathcal{N}^{j}} V(A)-V\left(A_{p \downarrow}\right) \\
& =\sum_{A \in \bigcup_{t=1}^{j^{n-1}} \mathcal{N}_{p}^{j}(t)} V(A)-V\left(A_{p \downarrow}\right) \\
& =\sum_{t=1}^{j^{n-1}} \sum_{A \in \mathcal{N}_{p}^{j}(t)} V(A)-V\left(A_{p \downarrow}\right) .
\end{aligned}
$$

For any $t$, the cardinality of $\mathcal{N}_{p}^{j}(t)$ is $j$, and

$$
\sum_{A \in \mathcal{N}_{p}^{j}(t)} V(A)-V\left(A_{p \downarrow}\right)=V\left(A_{p}^{1}(t)\right)-V\left(A_{p}^{j}(t)\right)
$$

where $A(t)$ is any representative $j$-partition in the class $\mathcal{N}_{p}^{j}(t)$. Thus,

$$
\begin{aligned}
\eta_{p}(V) & =\sum_{t=1}^{j^{n-1}} \sum_{A \in \mathcal{N}_{p}^{j}(t)} V(A)-V\left(A_{p \downarrow}\right) \\
& =\sum_{t=1}^{j^{n-1}} V\left(A_{p}^{1}(t)\right)-V\left(A_{p}^{j}(t)\right)
\end{aligned}
$$

On the other hand,

$$
\begin{aligned}
& \sum_{A \in \mathcal{N}^{j}} V\left(A_{p}^{1}\right)-V\left(A_{p}^{j}\right) \\
= & \sum_{A \in j_{t=1}^{n-1}} V\left(A_{p}^{1}\right)-V\left(A_{p}^{j}\right) \\
= & \sum_{t=1}^{j^{n-1}} j\left(V\left(A_{p}^{1}(t)\right)-V\left(A_{p}^{j}(t)\right)\right) \\
= & j \sum_{t=1}^{j n-1} V\left(A_{p}^{1}(t)\right)-V\left(A_{p}^{j}(t)\right) \\
= & j \eta_{p}(V)\left(\text { from the expression of } \eta_{p}(V) \text { above }\right) .
\end{aligned}
$$

It follows that:

$$
\eta_{p}(V)=\frac{1}{j} \sum_{A \in \mathcal{N}^{j}} V\left(A_{p}^{1}\right)-V\left(A_{p}^{j}\right)
$$

A simple application is provided below.

Example 1. A monotonic hierarchical organization $(N, T, V)$ involves two workers $p$ and $q$ and four tasks (the tasks may also be viewed as different levels of effort). All the possible allocations of workers are given in the first column of the table below. Note that we write $(p, \varnothing, \varnothing, q)$, for instance, for $(\{q\}, \varnothing, \varnothing,\{q\})$, which denotes a configuration in which $p$ and $q$ are assigned to tasks $t_{1}$ and $t_{4}$, respectively, and no worker is assigned to task $t_{2}$ or task $t_{3}$. The production function of the organization is defined in the second column. The difference between the marginal productivity at the top level and at the bottom level of the hierarchy is given in the third column for worker $p$ and in the fourth column for worker $q$.

\begin{tabular}{cccc}
\hline 4-Partition (A) & $V(A)$ & $V\left(A_{p}^{1}\right)-V\left(A_{p}^{j}\right)$ & $V\left(A_{q}^{1}\right)-V\left(A_{q}^{j}\right)$ \\
\hline$(\varnothing, \varnothing, \varnothing, p q)$ & 0 & 0 & 0 \\
$(\varnothing, \varnothing, p, q)$ & 0 & 0 & 1 \\
$(\varnothing, \mathrm{p}, \varnothing, \mathrm{q})$ & 0 & 0 & 1 \\
$(\mathrm{p}, \varnothing, \varnothing, \mathrm{q})$ & 0 & 0 & 2 \\
$(\varnothing, \varnothing, \mathrm{q}, \mathrm{p})$ & 0 & 1 & 0 \\
$(\varnothing, \mathrm{q}, \varnothing, \mathrm{p})$ & 0 & 1 & 0 \\
$(\mathrm{q}, \varnothing, \varnothing, \mathrm{p})$ & 0 & 2 & 0 \\
$(\varnothing, \varnothing, \mathrm{pq}, \varnothing)$ & 0 & 1 & 1 \\
$(\varnothing, \mathrm{p}, \mathrm{q}, \varnothing)$ & 1 & 1 & 1 \\
$(\mathrm{p}, \varnothing, \mathrm{q}, \varnothing)$ & 1 & 1 & 2 \\
$(\varnothing, \mathrm{q}, \mathrm{p}, \varnothing)$ & 0 & 1 & 1 \\
$(\mathrm{q}, \varnothing, \mathrm{p}, \varnothing)$ & 1 & 2 & 1 \\
$(\varnothing, \mathrm{pq}, \varnothing, \varnothing)$ & 1 & 1 & 1 \\
$(\mathrm{p}, \mathrm{q}, \varnothing, \varnothing)$ & 1 & 1 & 2 \\
$(\mathrm{q}, \mathrm{p}, \varnothing, \varnothing)$ & 1 & 2 & 1 \\
$(\mathrm{pq}, \varnothing, \varnothing, \varnothing)$ & 2 & 2 & 2 \\
\hline
\end{tabular}

The Banzhaf score of each of the two workers is therefore $16 / 4=4$, which implies that the normalized Banzhaf value is $1 / 2$ for each. So each is expected to have half of the total output or revenue.

\section{Conclusion}

We have studied the distribution of earnings in a newly defined model of hierarchical organizations. Earnings are measured by the generalized Banzhaf value proposed by Freixas [1]. A new interpretation of this sharing rule has been given. More precisely, in any hierarchical organization, the normalized Banzhaf value of a worker is proportional to the difference between his marginal productivity at the top level and at the bottom level of the hierarchy summed over all the possible configurations of the organization. This new formulation is also easy to compute. 


\section{REFERENCES}

[1] J. Freixas, "The Banzhaf Measures for Games with Several Levels of Approval in the Input and Output," Annals of Operations Research, Vol. 137, No. 1, 2005, pp. 45-66. doi:10.1007/s10479-005-2244-9

[2] R. Pongou, B. Tchantcho and N. Tedjeugang, "On-theJob Trial Tournament: Productivity Rank and Earnings,” Mimeo, 2012.

[3] J. Banzhaf, “Weighted Voting Doesn't Work: A Mathematical Analysis,” Rutgers Law Review, Vol. 19, No.2, 1965, pp. 317-343.

[4] J. Coleman, "Control of Collectivities and the Power of a Collectivity to Act,” In: B. Lieberman, Ed., Social Choice, Gordon and Breach, New York, 1971, pp. 269-300.

[5] P. Dubey and L. Shapley, "Mathematical Properties of the Banzhaf Power Index," Mathematics of Operations Research, Vol. 4, No. 2, 1979, pp. 99-131. doi:10.1287/moor.4.2.99

[6] A. Laruelle and F. Valenciano, "Shapley-Shubik and Banzhaf Indices Revisited," Mathematics of Operations Research, Vol. 26, No. 1, 2001, pp. 89-104. doi:10.1287/moor.26.1.89.10589

[7] D. Leech, "The Relationship between Shareholding Concentration and Shareholder Voting Power in British Companies: A Study of the Application of Power Indices for Simple Games,” Management Science, Vol. 34, No. 4, 1988, pp. 509-529. doi:10.1287/mnsc.34.4.509

[8] D. Leech, “Computing Power Indices for Large Voting
Games,” Management Science, Vol. 49, No. 6, 2003, pp. 831-838. doi:10.1287/mnsc.49.6.831.16024

[9] V. Feltkamp, "Alternative Axiomatic Characterizations of the Shapley and Banzhaf Values,” International Journal of Game Theory, Vol. 24, No. 2, 1995, pp. 179-186. doi:10.1007/BF01240041

[10] J. Freixas and W. S. Zwicker, "Weighted Voting, Abstention, and Multiple Levels of Approval," Social Choice and Welfare, Vol. 21, No. 3, 2003, pp. 399-431. doi:10.1007/s00355-003-0212-3

[11] L. Diffo Lambo and J. Moulen, "Ordinal Equivalence of Power Notions in Voting Games," Theory and Decision, Vol. 53, No. 4, 2002, pp. 313-325. doi:10.1023/A:1024158301610

[12] J. Freixas, D. Marciniako and M. Pons, "On the Ordinal Equivalence of the Johnston, Banzhaf and Shapley Power Indices," European Journal of Operation Research, Vol. 216, No. 2, 2011, pp. 367-375. doi:10.1016/j.ejor.2011.07.028

[13] B. Tchantcho, L. Diffo Lambo, R. Pongou and B. Mbama Engoulou, "Voters' Power in Voting Games with Abstention: Influence Relation and Ordinal Equivalence of Power Theories," Games and Economic Behavior, Vol. 64, No. 1, 2008, pp. 335-350. doi:10.1016/j.geb.2007.10.014

[14] R. Pongou, B. Tchantcho and L. Diffo Lambo, "Political Influence in Multi-Choice Institutions: Cyclicity, Anonymity, and Transitivity," Theory and Decision, Vol. 70, No. 2, 2011, pp. 157-178. doi:10.1007/s11238-010-9211-X 